\title{
JOSÉ PEDRO VARELA Y LA GESTACIÓN DE LA ESCUELA URUGUAYA
}

\author{
Jorge Bralich ${ }^{1}$ \\ Universidad de la República - Uruguay \\ jbrassi@adinet.com.uy
}

Recepción: 28/03/2011

Evaluación: 09/05/2011

Aceptación: 22/10/2011

Artículo de Reflexión

\section{RESUMEN}

Nos proponemos destacar la importancia de José Pedro Varela en el concierto latinoamericano, como constructor del sistema escolar uruguayo. Este trabajo resume largas investigaciones sobre la obra y personalidad de Varela, realizadas años atrás y sintéticamente expresadas en nuestra obra "Varela" (1989). Dicha investigación se basó sustancialmente en la re-lectura de los escritos de aquel y la contextualización de los mismos en el ambiente social, político y económico en que vivió. De ello surgió que la imagen popularizada de Varela ha sido muy esquemática y no rescata lo complejo de su personalidad. En efecto, Varela, joven inteligente y dinámico, inspirado en la obra de Sarmiento, junto con varios jóvenes amigos impulsó la creación de un movimiento educacional (la "Sociedad de Amigos de la Educación Popular”) que confluyó en la aprobación -por el Gobierno- de una Ley de Educación y en su designación como Inspector Nacional de Instrucción Pública en 1877. Su temprana muerte en 1879 no le impidió dejar sentada las bases de un sistema escolar, cuyas líneas generales se prolongarían a través de más de un siglo. Si bien no ha sido suficientemente reconocido a nivel internacional, en Uruguay -y en alguna medida, en el Río de la Plata- es una de las figuras destacadas de la historia educacional.

Palabras clave: Revista Historia de la Educación Latinoamericana, Uruguay, historia de la educación, Varela.

1 Profesor honorario de la Facultad de Humanidades y Ciencias de la Educación adscrita al Departamento de Filosofía e Historia de la Educación. Ex-profesor/investigador en temas de historia de la educación uruguaya (jubilado) de la Facultad de Ciencias Sociales, Universidad de la República (Uruguay). Presidente de la Sociedad Uruguaya de Historia de la Educación (SUHE). 


\title{
JOSÉ PEDRO VARELA IN THE GESTATION OF THE URUGUYAN SCHOOL
}

\author{
Jorge Bralich \\ Universidad de la República - Uruguay \\ jbrassi@adinet.com.uy
}

\begin{abstract}
We propose to emphasize the importance of Jose Pedro Varela in the Latin-American concert, as builder of the Uruguayan school system. This work summarizes long researches about the work and personality of Varela, these were done years before and synthetically expressed in our work "Varela " (1989). The mentioned research was based on the re-reading of his writings their different contexts in the social, political and economic environment in which he lived. From it, it arose that the well-known image of Varela has been very schematic and it does not rescue the complexity of his personality. In effect Varela, an intelligent and dynamic young person inspired by the work of Sarmiento, together with several young friends stimulated the creation of an educational movement (the "Friends' Society of the Popular Education ") it came together in the approval - for the Government - of a Law of Education and in his designation as National Inspector of Public Instruction in 1877. His early death in 1879 did not prevent him from stopping laid the foundations of a school system, which general lines would extend across any more than one century. Though he has not been sufficiently recognized worldwide, in Uruguay - and in any measure, in the Rio de la Plata - it is one of the figures distinguished from the educational history.
\end{abstract}

Key words: Journal of Latin American Education History, Uruguay, bistory of education, Varela.

\section{JOSÉ PEDRO VARELA E A GESTAÇÃO DA ESCOLA URUGUAIA}

\author{
Jorge Bralich \\ Universidad de la República - Uruguay \\ jbrassi@adinet.com.uy
}

\section{RESUMO}

Propomo-nos a destacar a importância de José Pedro Varela no contexto latino-americano, como construtor do sistema escolar uruguaio. Este trabalho resume grandes pesquisas sobre a obra e personalidade de 
Varela, realizadas anos atrás e sinteticamente divulgadas em nossa obra "Varela" (1989). Esta pesquisa se baseou substancialmente na releitura dos escritos deste autor e na contextualização dos mesmos no ambiente social, político e econômico em que viveu. Neste trabalho concluímos que a imagem popularizada de Varela foi muito esquemática e não resgata completamente sua personalidade. De fato, Varela, jovem, inteligente e dinâmico, inspirado na obra de Sarmiento, junto com vários jovens amigos, impulsionou a criação de um movimento educacional (a "Sociedade de Amigos da Educação Popular") que confluiu na aprovação - pelo Governo - de uma Lei de Educação e em sua designação como Inspetor Nacional de Instrução Pública em 1877. Sua morte precoce em 1879 não lhe impediu de fixar as bases de um sistema escolar, cujas linhas gerais se prolongariam através de mais de um século. A despeito de não ter sido suficientemente reconhecido em nível internacional, no Uruguai - e em alguma medida, no Rio da Prata - é uma das figuras de destaque na história educacional.

Palavras-chave: Revista História da Educação Latino-americana, Uruguai, História da Educação: Varela.

\section{INTRODUCCIÓN}

\section{Varela en el contexto latinoamericano del siglo XIX.}

La presencia de José Pedro Varela en el concierto de los educadores latinoamericanos se justifica plenamente. No puede desconocerse que el sistema escolar uruguayo -que en el siglo XX fue considerado casi como ejemplar- surgió en el siglo XIX de sus impulsos de joven inteligente y trabajador, que supo interpretar adecuadamente las necesidades de una sociedad que se enfrentaba a un proceso de modernización, y supo también aprovechar las capacidades de un núcleo de jóvenes -como él, inteligentes y emprendedores- que deseaban transformar la sociedad en que vivían. Lo que sigue es apenas un esbozo de la obra que emprendió, de su contexto social y económico y de sus caracteres fundamentales.

Este trabajo resume largas investigaciones sobre la obra y personalidad de Varela, realizadas años atrás y sintéticamente expresadas en nuestra obra citada en la bibliografía. Dicha investigación se basó sustancialmente en la re-lectura de los escritos de aquel y la contextualización de los mismos en el ambiente social, político y económico en que vivió. Se partió de la hipótesis de que su figura se había convertido en mito nacional y -por lo tanto- no podía verse en su verdadera dimensión humana e intelectual. Muchísimos datos que aportaban luz a este proceso de reforma escolar había sido inconcientemente escamoteados por el afán de reafirmar su papel de ídolo 
nacional. Los referidos datos -empero- estaban al alcance de cualquiera que revisara la prensa de la época, o la correspondencia personal disponible en los archivos del Museo Pedagógico que lleva su nombre.

Un trabajo como el presente -necesariamente acotado en su extensiónno permite un desarrollo de toda esa rica realidad cultural, pero al menos permite vislumbrar algo más complejo que los esquemas históricos que se han difundido del reformador-constructor de la escuela uruguaya, desde la mirada de la historia social de la educacion.

\section{La forja de un educador: su juventud, sus viajes, sus contactos}

Pedro José Varela -luego José Pedro- nació el 19 de marzo de 1845 en un hogar de la burguesía comercial rioplatense; su padre -Jacobo Dionisioera un importante comerciante argentino, emigrado a Montevideo por razones políticas y con un buen bagaje cultural (había efectuado la traducción al español de una obra didáctica francesa: "La enseñanza de la lengua materna" del Padre Girard que, según parece, fue el primer libro de pedagogía editado en el Río de la Plata).La madre de José Pedro fue Benita Gumersinda Berro, sobrina de Dámaso Antonio Larrañaga, sacerdote de destacada actuación en la historia nacional. Fueron sus tíos por parte de padre: Florencio y Juan de la Cruz Varela, periodista y poeta respectivamente y por parte de madre: Adolfo Berro, poeta y Bernardo Prudencio, poeta que llegaría a Presidente del Uruguay en 1860. Si agregamos a este entorno primario, las vinculaciones posteriores surgidas de matrimonios (una hermana de José Pedro -Elvira- se casará con Alfredo Vásquez Acevedo -futuro rector de la Universidad, y compañero de andanzas de José Pedro-; un hermano -Jacobo Adrián- contraerá enlace con una hermana de Alfredo Vásquez, en tanto José Pedro se casará con una hija de Eduardo Acevedo -jurisconsulto, ministro, periodista-) tenemos un entorno familiar que "constituía una singular selección de valores sociales, morales e intelectuales. Mujeres de excepcional cultura, jurisconsultos, hombres de Estado, literatos, periodistas, comerciantes, hombres de pensamiento y acción (que) formaban aquel núcleo de familias, representativas de una clase social...".

El joven José Pedro no cursó estudios superiores, pero -acorde con su actividad en la barraca de su padre- hizo estudios comerciales en el Colegio de los Hermanos Escolapios. A los 19 años ya el joven Varela es una incipiente personalidad literaria, aunque naturalmente no sea el único en su generación. Comparte con un grupo de jóvenes amigos (los hermanos Ramírez: Gonzalo,

2 MONTERO BUSTAMANTE, R. (1922): "Jacobo Varela. Su vida y su obra".

Montevideo, Imprenta Mercantil, p. 67. 
Carlos y Octavio, Agustín de Vedia, Julio Herrera y Obes) sus inquietudes literarias, intercambiándose abundantes cartas y ensayos poéticos, que son comentados, elogiados y criticados con gran entusiasmo. En el lapso que transcurre desde su adolescencia hasta ese momento, parece haber ocurrido algún hecho que conmovió profundamente su espíritu aunque el mismo puede haber sido una normal crisis de adolescencia, hipertrofiada por el clima romántico de esa época en la que los jóvenes burgueses acostumbraban a convivir con la muerte, con el dolor, con la tristeza. A pesar de esa aura generalizada entre los jóvenes románticos, no era José Pedro un muchacho triste, por el contrario, en medio de sus románticas melancolías, surgen brotes de humor, de picardía, junto con manifestaciones de vanidad acordes con su destacada actuación en el grupo. Muy joven aún -con apenas 20 añosejerció el periodismo a través de una revista que fundó con algunos de sus jóvenes amigos: "La Revista Literaria", en la cual, además de poemas y notas de actualidad, publicó artículos sobre temas sociales y educacionales. En uno de estos artículos señalaba:

No necesitamos poblaciones excesivas, lo que necesitamos, es poblaciones ilustradas.

El día en que nuestros gauchos supieran leer y escribir, supieran pensar, nuestras convulsiones politicas desaparecerían quizás.

Es por medio de la educación del pueblo que hemos de llegar a la paz, al progreso y a la extinción de los gauchos.

Entonces, el habitante de la campaña a quien hoy embrutece la ociosidad, dignificado por el trabajo, convertiría su caballo, boy elemento de salvajismo, en elemento de progreso y trazaría con él, el surco que ba de hacer productiva la tierra que permanece hasta boy estéril.

$Y$ las inmensas riquezas nacionales movidas por el brazo del pueblo, trabajador e ilustrado, formarán la inmensa pirámide del progreso material.

La ilustración del pueblo es la verdadera locomotora del progreso ${ }^{3}$.

Fueron varios los artículos aparecidos en esta revista sobre el tema de la educación popular, escritos por: "Violeta" (seudónimo de una educadora argentina), Juana Manso (también argentina), Amadeo Jacques (francés), además de los colaboradores nacionales. En algunas de esas notas ya se destacan la figura y obra de Sarmiento que Varela conocerá al poco tiempo.

Poco después, en 1867, partió para Europa y EE.UU., en un viaje que era al mismo tiempo de formación cultural y de negocios. De ese viaje nos quedaron las cartas que publicó regularmente en el periódico El Siglo, en las que nos dio sus impresiones de los países visitados y de los contactos realizados.

${ }^{3}$ VARELA, J.P. (1865): “Los gauchos” en: La Revista Literaria. No 13, p. 207. 
En Europa se entrevistó con Víctor Hugo a quien le mostró sus poemas buscando sus comentarios, pero más significativos fueron sus contactos en EE.UU. en donde conoció personalmente a Domingo F. Sarmiento y Bartolomé Mitre, quienes entusiasmaron al joven Varela con la necesidad de que dedicase sus esfuerzos a transformar el sistema escolar uruguayo.

Abí está su campo (habría dicho Sarmiento a Varela). En nada podrá Ud. trabajar con más gloria y haciendo mayor bien. Estudié estos sistemas tan perfeccionados, imprégnese de la esencia americana, del mecanismo escolar, desde la renta hasta la banca y lleve a su país, que lo que necesita son ciudadanos aptos para desempeñar los deberes de tales, esta base inconmovible de engrandecimiento nacional. "Llenaría páginas y más páginas si bubiera de condensar y a que no repetir, sus consejos a Varela sobre esta materia". "Yo también metí mi cucharada en la catequización del joven Varela, que debió pensar que habia caído en una casa de locos atacados de la manía sajona, de tal modo le metíamos por los ojos libros y diarios ingleses, le elogiábamos cosas inglesas y le pintábamos magnificos horizontes ingleses ${ }^{4}$.

A estas sugerencias de los educadores argentinos, se agregó el impacto que experimentó el joven viajero ante el desarrollo económico, social y político de EE.UU; como -por ejemplo- la destacada actuación pública de algunas mujeres en el plano político.

Varela vio entonces definirse más claramente su vocación de educador, volviendo a Uruguay con la firme decisión de emprender una tenaz campaña en pro de la escuela pública.

\section{El contexto social y económico de Uruguay en la segunda mitad del siglo XIX: El estado de las escuelas primarias, los reclamos sociales, los ensayos educacionales}

El período que va desde la Declaratoria de la Independencia -1825- hasta fines de los 60, estuvo jalonado por una sucesión continua de luchas entre grupos nacionales, a veces con el apoyo de potencias extranjeras. Entre los más de 40 levantamientos contra las autoridades de turno, se destacan por su trascendencia: la Guerra Grande -extendida por más de 10 años- y la Cruzada Libertadora -de 1863 a 1865- que ocasionó más de 1000 muertos entre ambos bandos. Pese a ello los distintos gobiernos intentaron desarrollar la educación

4 VÁZQUEZ ROMERO, A. (1969): “José P. Varela”. Montevideo.1979. Carta de Mitre y Vedia a Sarmiento, en: Revista Histórica. $N^{o} 118-120$. Montevideo. p.13. Ver: JARDILINO, Rubens, PROVENCA, Leandro. (2011): "Sarmiento y Varela. Caminos de cruces en la Educación del cono Sur en el siglo XIX", en: Educadores en América Latina y el Caribe. De la Colonia a los siglos XIX y XX. Colección Educadores, Tomo 3, pp. 123-144. 
pública, considerando que esa podía ser una vía de pacificación y civilización. Con ilusiones, se dictaron leyes y decretos que pretendían organizar e impulsar el sistema educativo público: Ley de Educación de 1826, creación del Instituto de Instrucción Pública, etc. En 1855, un informe redactado por el Secretario de este Instituto de Instrucción Pública -conocido como "Informe Palomeque"- señalaba, entre otras cosas:

El desquicio, la inercia, la falta de sistema y de medios y el desconocimiento de los reglamentos de estudios, es la guía, la base, la uniformidad y suficiencia con que se pretende educar a nuestras poblaciones atrasadas. Cuando se babla de educación en los departamentos de campaña se dice una mentira o se inicia una farsas.

Resulta también del informe que, para una población de 129.000 personas en los 11 departamentos visitados, sólo asistían 899 escolares en 30 escuelas, en tanto que las propuestas efectuadas por el Cnel. Palomeque tendientes a comenzar una reorganización del sistema escolar no fueron atendidas por el gobierno, aduciendo falta de recursos.

La necesidad de un sistema educativo nacional se hacía necesaria además por el empuje inmigratorio que desde 1835 golpeaba nuestro territorio haciendo -por ejemplo- que en 1843 hubiese en Montevideo una mayoría de extranjeros (19.252 frente a 11.431 orientales) y que en 1860 hubiese en todo el país un $25 \%$ de extranjeros. El casi inexistente sistema educacional no logró integrar a esa masa inmigrante, que -por el contrariomodeló en gran medida la cultura nacional.

Los reclamos acerca de esa imprescindible reorganización eran señalados con firmeza por varios sectores de la sociedad. En 1871 se constituyó la Asociación Rural del Uruguay (A.R.U.) para nuclear a los hacendados progresistas, aquellos que abandonando la explotación casi salvaje de la ganadería, optaban por una explotación racional. Esto último implicaba el mestizaje del ganado vacuno, la introducción del ovino la vacunación de los animales, el cercamiento de los campos, etc. lo que requería necesariamente un cambio en las formas de trabajo agrario, menos brutal, más disciplinado. Estos hacendados en su revista dedicaron numerosos artículos al tema educacional, en los que exigían una mejora de las escuelas de campaña.

La educación, base fundamental de la existencia y progreso de las sociedades, no ha llegado aún a sus poblados campos... "se dirá en el editorial del $\mathrm{N}^{\circ} 1$ de esa revista ${ }^{6}$ y más adelante, en otro artículo se expresará:

5 ARAÚJO, O. (1911): "Historia de la escuela Uruguaya". Montevideo, El siglo ilustrado, pp. 330-346.

6 CASTELLANOS, José Ma. (1872): Revista de la ARU. Editorial del No.1.15/ mar/1872. 
¿Cómo no pedir a gritos, escuelas, escuelas y escuelas, para todos los bijos desheredados del desierto? Empecemos por ese camino a formar el ciudadano. La escuela es la cuna del patricio suizo y americano que despiertan nuestra envidia y que admiramos... Que la primera y más crecida cifra de nuestro presupuesto sea para la educación, extendida y ramificada por todas partes. Obligatoria para todos los que nacen en nuestro territorio; llevada al rancho por la mano del Estado que más tarde le ha de pedir al ciudadano su saber y su sangre. Educación fundada en la moral del Evangelio y el trabajo; que al mismo tiempo se enseñe a comprender los deberes, como a satisfacer las necesidades por medio del trabajo; que se sepa, en fin, labrar la tierra, como manejar el lazo y la pluma y leer el Evangelio...7

Las exigencias de estos hacendados iban, pues, hacia la necesidad de un cambio no sólo en las modalidades de trabajo, sino también, en las costumbres y usos sociales, radicando al gaucho con su familia y haciendo de él un agricultor tanto como un ganadero. A estos reclamos debemos agregar también los que provenían de otro sector bastante alejado del mencionado: los obreros, principalmente los de origen europeo que traían de aquel continente ideas avanzadas respecto a la organización social. Buena parte de la inmigración que llegaba desde Europa estaba constituida por trabajadores que había vivido ya experiencias de luchas sociales, incluso revolucionarias.

...en la sociedad presente... el trabajador, el ser productor por excelencia, es burlado y explotado en sus más justas aspiraciones. Para él la ciencia, ese alimento intelectual, es una palabra. Y sin embargo, él es la base de esa misma ciencia; sin su trabajo, los hombres que se dedican a cultivarla no podrían hacerlo... El tiempo tan necesario para el descanso, la instrucción y el recreo le es quitado también... ¿Que coma, vista y se instruya pésimamente, el que crea y transforma la riqueza social!' QQue todo lo acapare y lo goce el que nada produce! ${ }^{8}$.

El primer factor del progreso es la instrucción. ¿Está el proletario en condiciones de instruirse, cuando por atender a su subsistencia y a la de su familia tiene que emplear por término medio 120 ó más horas de trabajo para conseguirlo?... El hombre no puede ser libre si no es instruido, de aquí que las batallas todas del proletariado de boy deben concretarse a aminorar sus horas de trabajo.

A todas estas demandas de reformas educativas, debemos agregar la presión ejercida por un importante sector de jóvenes intelectuales, que -liderados en parte por Varela- comenzaron a experimentar con nuevas

7 GÓMEZ, Juan Ramón. (1873): Revista ARU, 15 de septiembre.

8 RAMA, Carlos. (1969): “Obreros y anarquistas". Enciclopedia Uruguaya No. 32. (Trascripción de un folleto de la Asociación Internacional de Trabajadores de Montevideo. Montevideo. 1875). Edit. Arca.

9 Periódico EL TIPÓGRAFO. (1884). Nota del 16 de enero. 
formas educacionales. En efecto, a su regreso del viaje por Europa y EE.UU. -en septiembre de 1868- Varela dio una conferencia en los salones del Instituto de Instrucción Pública, en la cual desarrolló las ideas sobre educación que había ido moldeando desde su encuentro con Sarmiento:

Desquiciada la sociedad, abandonada la industria, embrutecidas las masas, desbordadas laspasiones, el cuadro quepordoquiera ofrecen las Repúblicas sudamericanas es desconsolador, desesperante.. ¿Qué le falta a la América del Sur para ser asiento de naciones poderosas? Digámoslo sin reparo: instrucción, educación difundida en la masa de sus habitantes, para que sea cada uno elemento y centro de producción y de riqueza, de resistencia inteligente contra los bruscos movimientos sociales, de instigación y freno al gobierno ${ }^{10}$.

La prensa recogió ese hecho y lo comentó de esta manera: Como lo anunciáramos tuvo lugar la noche del viernes transcurrido la conferencia iniciada con el objeto de cambiar algunas ideas a propósito de una lectura presentada por nuestro ilustrado compatriota JoséPedro Varela. Los salones de la Universidad encerraban una concurrencia que creemos excedía a 300 personas... La lectura fue brillantísima, siendo saludada con vivos aplausos por los concurrentes ${ }^{11}$.

A la intervención de Varela siguió la de otro de sus compañeros de ideas -Carlos María Ramírez- que expresó:

La Sociedad de Amigos de la Educación Popular podrá decir a los estancieros: dad una parte de vuestras rentas para la educación del pueblo, porque si el pueblo se educa, mañana no estarán en él los elementos de la montonera que harán depreciar el valor de vuestros campos, que talará vuestros sembrados, que acabarán con vuestra hacienda. La Sociedad de Amigos de la Educación Popular podrá decir a los propietarios de la ciudad: dad una parte de vuestra renta para la educación del pueblo en la ciudad y en la campaña, porque si el pueblo no se educa, mañana las masas ignorantes y semisalvajes se lanzarán al exterminio contra las clases civilizadas y egoistas. La Sociedad de Amigos de la Educación Popular podrá decir a los comerciantes todos: dad una parte de vuestra renta para educar el pueblo, porque si el pueblo no se educa, vuestro comercio llevará una vida precaria y miserable bajo la amenaza continua de los trastornos políticos, de las rebeliones $y$ las guerras. Y podrá decir al pueblo entero: vamos a reunir nuestros esfuerzos para educarnos a nosotros mismos, porque sin la educación no bay trabajo, ni riqueza, ni adelanto, no bay derecho de ciudad en la República, ni derecho de civilización en la bumanidad ${ }^{12}$.

${ }^{10}$ VÁZQUEZ ROMERO, Andrés. (1979): "José Pedro Varela". Montevideo. p.56.

${ }^{11}$ Diario "La Tribuna", 20 septiembre.

${ }^{12}$ Revista "LA EDUCACIÓN POPULAR" (Prospecto) Soc. de Amigos de la Educ. Popular (Bibl. Central de Enseñanza Secundaria). 
Luego de estas intervenciones se resolvió constituir una "Sociedad de Amigos de la Educación Popular" (S.A.E.P.) que desarrolló a partir de ese momento una intensa obra educacional: fundación de escuelas, bibliotecas populares, publicación de obras pedagógicas, cursos para maestros, etc. Todos estos emprendimientos tuvieron como respaldo pedagógico no solamente el aporte personal de quienes integraban la sociedad, sino también el rico bagaje de información que Varela había traído de EE.UU. conformado por varios cajones con literatura pedagógica originaria de aquel país (entre otras obras, el "Journal of Education" de Barnard, importante publicación enciclopédica). Cabe señalar también, que muchos de los que participaron en las actividades de esta sociedad desde 1868, integraron luego la Asociación Rural que más arriba mencionábamos, lo que muestra que este movimiento no era producto del simple entusiasmo de un grupo de jóvenes, sino que respondía a las inquietudes de varios sectores de la sociedad.

La SAEP puede ser considerada promotora del proceso de reforma educacional vareliana aunque no fue obra exclusiva de Varela. Si bien él tuvo un papel protagónico en sus comienzos y participó activamente durante toda su vida, el núcleo de hombres jóvenes que lo rodeaban, no eran meros seguidores dóciles de sus ideas e iniciativas, sino que por si mismos, eran gestores de nuevas ideas y activos realizadores, incluyéndose en este grupo figuras que más tarde adquirirían un relieve tan importante como el de José Pedro Varela: su propio hermano Jacobo, Alfredo Vásquez Acevedo, Carlos Ma. Ramírez, Francisco A. Berra, Emilio Romero, etc.

Otro antecedente de la reforma educativa a considerar fue el proyecto de Ley de Educación presentado por Agustín de Vedia, en 1873. Este constaba de 77 artículos, que abarcaban todos los aspectos del sistema escolar.

Respecto a la estructura general del sistema, se inclinaba -como lo señala en su fundamentación- por la descentralización estableciendo Juntas Vecinales de instrucción pública, compuestas por 3 miembros, electos por el voto popular (que incluía a los extranjeros que pagaban contribución directa) las cuales podrían: dictar reglamentos, supervisar la enseñanza y los inspectores, promover la asistencia escolar (pero sin hacerla obligatoria). Los inspectores, por su parte, administraban los recursos escolares, creaban o subdividían escuelas, nombraban y destituían a los maestros y ayudantes.

La ley creaba también colegios de enseñanza superior -equivalente a los primeros años de la actual enseñanza media- en cada cabeza de departamento, escuelas nocturnas para adultos, dos escuelas normales y bibliotecas populares anexas a cada escuela. Por otra parte establecía recursos especiales para la educación, destinando a la misma el 5 o/oo de la Contribución Directa; establecía la entrega de libros de texto y útiles 
en forma gratuita, becas en dinero para los estudiantes de magisterio que provengan del interior del país, así como la exigencia de título habilitante para ejercer el magisterio.

Por último, además de establecer la libertad de estudios, instituía la prohibición de toda enseñanza religiosa en las escuelas públicas. Estas dos proposiciones dieron lugar -una vez presentado el proyecto en la Cámara y pasado el mismo a estudio de una Comisión de Legislación- a distintas reacciones: desde el apoyo que un núcleo de jóvenes universitarios dieron al proyecto de libertad de estudios, hasta el ataque violento de las jerarquías católicas. Las circunstancias políticas no permitieron que el proyecto se discutiese a fondo y al fin quedó sin aprobarse.

\section{La ideología de Varela: Su concepción de la sociedad uruguaya, la función de la escuela}

José P. Varela, al igual que muchos de sus compañeros de la Sociedad de Amigos de la Educación Popular, se vio arrastrado por el acontecer político. En 1869 fundó el diario "La Paz" periódico de oposición al gobierno de Lorenzo Batlle, en el que Varela continuó expresando los ideales políticos que ya había adelantado en "La Revista Literaria":

Queremos para la República el amor de sus hijos y el respeto del extranjero; para el ciudadano el goce de todos sus derechos y el cumplimiento de todos sus deberes; para el individuo, conciencia de sus actos y responsabilidad de sus acciones; para el hombre, en fin, la libertad dentro de los límites de la ley y para la ley la acción de la comunidad sobre el individuo, limite señalado al derecho individual por las más grandes aspiraciones de las sociedades modernas; para el extranjero, prosperidad y riqueza, a cambio de moralidad y trabajo; para el pueblo, educación que ensancha los horizontes del espiritu, llama a la vida activa todas las fuerzas del ser bumano y sirve de ancha y sólida base a toda felicidad, prosperidad y progreso... ${ }^{13}$.

Su campaña periodística a través de este diario le valió su destierro a Buenos Aires, de donde volvió en 1872, tras la revolución de 1870 y la subsiguiente firma de la paz. Empero, sus intentos de seguir militando políticamente se volvieron a frustrar tras los sangrientos sucesos que impidieron las elecciones para Alcalde, en la que Varela se postulaba. Las persecuciones políticas que se sucedieron forzaron a Varela a emigrar nuevamente a Buenos Aires, en donde funda otro periódico -opositor al gobierno uruguayo- y escribe una obra de carácter educacional: "La Legislación Escolar", en la cual, luego de analizar con dureza la situación

\footnotetext{
${ }^{13}$ VÁZQUEZ ROMERO, Andrés. (1979): Óp., cit, p.20.
} 
política de la sociedad uruguaya, propone una reforma del sistema educacional.

\section{Las ideas educacionales: Renovación metodológica y curricular}

La concepción vareliana dela educación está expuesta fundamentalmente en las dos obras mayores que publicó: "La educación del pueblo" y "La Legislación Escolar". La educación que propone Varela en la primera de estas obras es aquella que cumpla varias funciones: la erradicación de los males de la ignorancia (creencias supersticiosas de todo tipo: astrología, ánimas en pena, mal de ojo); la formación del obrero para trabajos calificados, por los que recibirá entonces mejor salario y aumentará su fortuna; la prolongación de la vida instruyendo sobre la conservación de la salud y ahorrando esfuerzos con el aprovechamiento de las nuevas técnicas; el aumento de la felicidad sustituyendo los placeres groseros de los ignorantes por el disfrute que da el conocimiento de la naturaleza, de la historia, de la humanidad; la disminución de los vicios y crímenes al mejorar las condiciones de vida de los pueblos y -por último- la contribución al progreso de las naciones, gracias a la instrucción de sus ciudadanos. Varela reafirmaba este último aspecto señalando: "El sufragio universal supone la conciencia universal y la conciencia universal supone y exige la educación universal"14.

Avanzaba -por otra parte- en cuales serían los contenidos y los métodos de la educación que él reclamaba. En los aspectos estrictamente pedagógicos, su propuesta se fundamentó en las corrientes cientificistas derivadas del positivismo, apoyándose en las ideas de Spencer a través de los pedagogos norteamericanos. En lo que respecta a los contenidos de la educación, Varela innova con respecto a lo que era usual en el medio nacional, planteando la necesidad de enseñar no sólo los contenidos básicos (lectura, escritura, cálculo) y algo de historia y geografía, sino además, introducir al niño en las ciencias naturales (física, fisiología, etc.) y cultivar su organismo mediante la gimnasia, el canto, etc. Pero lo que implicaba un paso aún más audaz, fue la introducción de las "lecciones sobre objetos", que Varela describe como:

simples lecciones orales, ordenadas y progresivas o conversaciones con los niños, haciéndoles observar y conocer los nombres, partes, propiedades, usos, etc. de los objetos más comunes, que fácilmente pueden presentarse a los niños o que son conocidos por ellos”. Y se extiende más adelante, diciendo: "Los colores, la forma y las partes prominentes de los objetos es lo que primero fija la atención de los

${ }^{14}$ VARELA, José Pedro. (1910): “La Educación del Pueblo”. Cap. VIII, El Siglo Ilustrado, p.55. 
niños y lo que más despierta su interés. Ver y tocar, es el anbelo constante de los niños. Es sólo por medio de los sentidos que se sienten impresionados y así la Naturaleza misma nos aconseja el uso de objetos sensibles para despertar su atención y evocar infantiles pensamientos. La vida intelectual de los niños está principalmente en las sensaciones y así el estado intelectual de la primera infancia exige objetos sensibles para poner la mente en actividad". "Estas lecciones sobre objetos, pueden extenderse fácilmente hasta dar con ellas un conocimiento sistemático de las ramas comunes de las ciencias experimentales ${ }^{15}$.

Así pues, la educación no debía limitarse a la acumulación de conocimientos en la mente del niño, sino extenderse al cultivo de habilidades intelectuales:

El pensar, en su más lato sentido, abraza toda la acción intelectual, desde el más simple acto de percepción hasta el vuelo atrevido de la imaginación, o el profundo proceder del raciocinio. Adquirir el modo de usar, de la mejor manera posible, todas las facultades intelectuales, es el fin primordial de la cultura mental. $Y$ parece obvio que el mejor medio de conseguir ese fin es practicar ejercicios, especialmente adaptados para guiar la mente al pensar ${ }^{16}$.

No fue Varela -por cierto- un pensador original en el plano pedagógico. El mismo lo asume así, diciendo en la introducción a su obra "La Educación del Pueblo":

Está lejos de ser una improvisación: este libro es el resultado de 6 $u 8$ años de estudios, seguidos con inalterable constancia a través de todos los acontecimientos de la vida, en el firme y decidido propósito de profundizar, hasta donde me fuese posible, las cuestiones relativas a la educación del pueblo y de buscar la verdad. $Y$ es esto tanto más cierto cuanto que ni remotamente aspiro a los bonores de la originalidad. Soy de los que creen que la educación es una verdadera ciencia, en cuyo campo solo puede uno agitarse con provecho, después de realizar detenidos y meditados estudios... ${ }^{17}$.

Y en oportunidad de la inauguración de las conferencias para maestros:

Fuera en mi insana pretensión, querer bacerlo, exponiendo ideas de mi propia cosecha, ya que no tengo títulos que a ello pudieran autorizarme, ni es posible, al exponer los límites de una ciencia, hacer otra cosa que seguir las huellas de los que han sabido

${ }^{15}$ VARELA, José Pedro. (1910): Cap. XIV-Ap. III.

${ }^{16}$ Ibídem., Cap. XIV-Ap. III.

${ }^{17}$ VARELA, José Pedro. (1910): Óp., cit., p.8. 
condensar el caudal atesorado por sucesivas generaciones de sabios y pensadores... ${ }^{18}$.

Acorde con esto, los trabajos pedagógicos de Varela recurren a frecuentes y abundantes transcripciones de obras de autores norteamericanos o ingleses: Lalor, Webster, Canning, Mayhew, y -fundamentalmente- Horacio Mann y es probable además, que muchas de las obras de los autores referenciados, hayan sido consultadas en los artículos del Journal of Education de Barnard, que Varela había adquirido en EE.UU. y no directamente en sus ediciones originales.

\section{La propuesta de Varela: la legislación escolar}

En abril de 1875 el Presidente Pedro Varela decretó que la Comisión de Instrucción Pública -dependiente de la Junta Económico-Administrativa del Departamento de Montevideo- asumiera la supervisión y dirección de todas las escuelas públicas del país, que hasta ese entonces estaban bajo la administración del Instituto de Instrucción Pública. De esta manera se daba la curiosa situación de que todas las escuelas del país pasasen a depender de una comisión de la Junta Económico-Administrativa de Montevideo.

El gobierno designó, a continuación, para presidir esa Comisión a José María Montero, íntimo amigo y colaborador de José P. Varela en su actividad educacional y política y -por otra parte- mano derecha del Cnel. Latorre, que a la brevedad sería Gobernador Provisorio de Uruguay. En los meses subsiguientes, Montero dio a las escuelas públicas un fuerte empuje: inauguración de nuevos locales, distribución de textos, concursos para maestros, etc. Esta labor fue reconocida por el propio José P. Varela, cuando poco después sustituye a Montero en dicho cargo, a propuesta del propio Montero, que pasa a ejercer como Ministro de Gobierno del Cnel. Latorre:

Es pues, bastante satisfactorio el estado actual de las escuelas públicas, en lo que se refiere a los locales, al menaje y a los útiles; y esta observación puedo hacerla con tanta más imparcialidad, cuanto que, en esta parte, casi todo estaba hecho cuando me recibi de la Dirección de Instrucción Pública sustituyendo a Montero, de modo que la actual Comisión apenas si ha tenido que ir perfeccionando en lo posible, las mejoras que babian sido introducidas por sus antecesores en el puesto. Ese mejoramiento... débese en gran parte a mi antecesor en el puesto, don J. M. Montero (bijo), quien puso especial empeño en obtener para las escuelas públicas, edificios espaciosos, bien situados y con las condiciones favorables que era posible obtener....

\footnotetext{
${ }^{18}$ Ibídem., p. 8.

${ }^{19}$ VARELA, José P. (1878): Memoria de la Dirección General. de Instrucción Primaria 1876-77.
} 
Al parecer, José P. Varela tuvo ciertas dudas antes de aceptar este cargo, ya que provenía de un gobierno de facto -el Gobernador Provisorio Cnel. Lorenzo Latorre- contra los que había luchado poco antes en aras de sus ideas políticas, pero -según habría expresado más tarde- consideraba que el beneficio que podía significar para el país una mejora de la escuela pública, era mayor que el prestigio que podía alcanzar el gobierno con esa mejora.

Una de las primeras acciones del nuevo Director de Instrucción Pública, fue presentar al Gobernador Latorre un proyecto de ley que se proponía organizar todo el sistema escolar de la república. En esa presentación, Varela decía:

Tengo el profundo convencimiento de que el Gobernador Provisorio realizaría un grande y fecundo esfuerzo en pro de la regeneración de la patria, si resolviese de una manera más o menos perfecta el problema de la organización de la enseñanza pública en nuestro pais, dándole bases sólidas y abriéndole un vasto campo de acción. No es dudoso que el fallo tranquilo e imparcial del historiador futuro lo juzgaría, si no como la más fecunda, como una de las más fecundas resoluciones tomadas por los gobiernos de la República, para responder a las necesidades más permanentes, más supremas y más vitales de la patria ${ }^{20}$.

Ese proyecto tomó la forma de un libro ("La Legislación Escolar") que ya hemos mencionado y en la primera parte del mismo -como fundamento de la ley proyectada- se analiza la situación del país de manera muy crítica, tanto en los aspectos políticos, como en los financieros, los culturales y específicamente los educacionales. En la segunda y tercera parte de esta obra se desarrolla el proyecto de organización educacional de manera pormenorizada y fundamentada.

En la descripción del sistema, Varela comienza por la base para facilitar así su mejor comprensión y -seguramente- para enfatizar el papel que pretende dar a la participación popular: "En vez de seguir el orden que naturalmente hemos debido seguir al formular el Proyecto de Ley, empezando por la Comisión Nacional de Educación, seguiremos un orden inverso y empezaremos por la fuente, es decir, por las Comisiones de Distrito"n.

Estas comisiones son la base del sistema: Varela previó la división de todo el sistema escolar en unidades básicas, denominadas distritos. Cada una de ellas abarcaba un sector de la población que no sobrepasaba los 500

${ }^{20}$ VARELA, José Pedro. (1964): La Legislación escolar. Biblioteca Artigas. Tomo. I. p. 14 .

${ }^{21}$ Ibídem., Tomo. II. p. 71. 
habitantes en el medio rural y en las ciudades podía llegar a 1000 ó 2000. Cada uno de estos distritos debía contar con -al menos- una escuela pública y al frente del sistema estaría una Comisión de Distrito integrada por tres vecinos, electos por todos los jefes de familia residentes en el distrito que supieran leer y escribir, inclusive aquellas mujeres que fueran también jefas de familia o extranjeros, siempre que abonasen Contribución Directa. Las potestades de estas comisiones eran amplias: designar y destituir los maestros (que debían ser titulados), establecer el programa y horario escolar (sobre la base de un programa mínimo establecido por las autoridades centrales), recaudar los fondos necesarios a través de un impuesto específico, alquilar o construir locales para las escuelas, etc. Los fondos necesarios para el mantenimiento de las escuelas provendrían de lo que recaudase cada Comisión de Distrito más una cantidad igual aportada por el Estado.

Los distritos escolares de una sección policial (en el medio rural) o una ciudad, conformarían una Sección Escolar, en tanto las secciones escolares de cada departamento del país, conformarían un Departamento escolar. Al frente de cada sección y departamento estaría un Inspector -de Sección o Departamental- el cual sería elegido por el voto de los distritos escolares. Asimismo, el Inspector Nacional, responsable de todo el sistema escolar, sería elegido también por el voto de los distritos.

Esta estructura del sistema escolar, desde la propia base contrastaba netamente con el pensamiento político vigente en esa época en esta región, en donde no sólo el centralismo era lo corriente, sino que -además- éste se veía reforzado por el autoritarismo de los gobiernos de fuerza que también eran moneda corriente. ¿Por qué esta propuesta tan a contra corriente de Varela? Simplemente porque éste se había inspirado largamente en el ejemplo de las escuelas de EE.UU., país que se había organizado sobre bases culturales y sociales distintas, en las que las comunidades tenían un desarrollo político mayor y un gran arraigo del valor "autonomía". Los fundamentos que ofrece Varela a su propuesta no dejan de ser acertados, en cuanto señala la importancia de la participación ciudadana para un buen resultado educacional:

Centralizada la administración escolar, la dirección superiorestará confiada sea a una persona como jefe de la instrucción pública, o sea a una corporación compuesta por varios miembros, pero forzosamente... la acción directa de ese centro tiene que hallarse limitada a una zona relativamente pequeña... La dirección tiene que limitarse a dictar reglas generales; programas de instrucción que persigan el quimérico sueño de ser igualmente adaptable a todas las escuelas; nombramientos de maestros para ir a regentear una escuela determinada, sin que se tenga conocimiento exacto de las necesidades de esa misma escuela... creación de escuelas arbitrariamente... y sobre todo esto, la inspiración de un hombre o de una corporación, modelando a su antojo la 
educación del pueblo y obligando a todas las inteligencias consagradas a esa tarea a encuadrarse de buena o mala gana, dentro de los límites arbitrariamente señalados por la Dirección... fácil es convencerse de la impotencia de una administración, por hábil y afanosa que sea... Cada localidad tiene su fisonomía propia, sus exigencias peculiares que solo pueden apreciarse bien, juzgando sobre el terreno, por los habitantes de la misma localidad... Y si esto es exacto ¿como podrá una administración centralizada, por activa e inteligente que sea, dar satisfacción a todas esas múltiples y complicadas exigencias de detalle?... ¿No es risible figurarse al Inspector de Instrucción Pública de Montevideo, ocupándose de resolver... si deben empezar las clases a las nueve o nueve y media, en una pequeña escuela rural de alguna de las secciones del Dep. de Maldonado? ¿Se concibe acaso que la autoridad central descienda a esos detalles, y aún si descendiese, que pudiera resolver con criterio, en cada caso particular, las mil pequeñas dificultades que en la organización de toda escuela se presentan? ${ }^{22}$.

Empero, no tenía en cuenta Varela el escaso desarrollo cultural y político de nuestra población, sobre todo en la campaña. Para ser elector y elegible de las Comisiones de Distrito, tenía que saber leer y escribir y la masa de la población rural y urbana jera analfabeta! Por otra parte, esas comisiones exigirían una dedicación que difícilmente quisieran asumir los vecinos, que no estaban acostumbrados al ejercicio de actividades de gobierno.

A la propuesta anterior, relativa al gobierno del sistema escolar, Varela agregó también otros aspectos, algunos de los cuales fueron también polémicos para el momento histórico. Si bien la gratuidad escolar no resultaba en absoluto inesperada, ya que las escuelas anteriores habían contemplado esa posibilidad para los niños pobres, las otras dos propuestas -obligatoriedad y laicidad- causaron asombro y protesta: la obligatoriedad escolar porque contradecía un aceptado derecho del padre de familia a enviar o no su hijo a la escuela y tal obligatoriedad implicaba avasallar su libertad. La otra propuesta merece una consideración especial: la población del país era mayoritariamente católica y esa era por lo tanto la religión oficial del Estado, de acuerdo con la Constitución. Varela, cercano por sus ideas a la masonería, rechazaba íntimamente el dogmatismo de esta Iglesia y pretendía una educación no dogmática:

¿En la escuela, la educación moral debe separarse de la enseñanza de las religiones positivas, o por el contrario, debe la educación general del individuo, tener por base la enseñanza dogmática?... ...vamos a sostener la justicia y la conveniencia de no enseñar en las escuelas públicas, o mejor dicho, de no enseñar en las escuelas los dogmas de una religión positiva cualquiera ${ }^{23}$.

${ }^{22}$ VARELA, José Pedro. (1964): Óp., cit, Tomo I, Cap. VIII, p.184.

${ }^{23}$ VARELA, José Pedro. (1964): Óp., cit, Tomo II, Cap. XI, p. 74. 
De todas maneras, al tener en cuenta -en este caso- las posibles resistencias sociales, busca una fórmula conciliatoria:

Nuestro razonamiento ha sido este: la mayoría de los habitantes de la República profesa la Religión Católica y cree que la escuela es hereje cuando en ella no se enseña el catecismo.., si se acuerda a las Comisiones de Distrito la facultad de establecer en la escuela la enseñanza del catecismo con ciertas restricciones, los católicos no tendrán causa para rechazar el sistema de educación común. Ahora bien: aún cuando nosotros creamos que es mejor la escuela pública en que no se enseña religión positiva alguna, creemos también que entre la escuela con catecismo y la carencia de escuela, es mejor lo primero que lo último... Cuando se legisla, cuando se toca tierra, hay que tomar en cuenta las sinuosidades y las asperezas del camino ${ }^{24}$.

La fórmula que propuso Varela fue que se enseñase la religión católica en el aula, pero si un solo padre se oponía a ello, esa enseñanza debía darse a los otros niños fuera de las horas de clase. Con esto pretendía contemplar el principio de la libertad de pensamiento y de creencia sin despertar demasiada oposición en la mayoría de la población que aceptaba la religión católica.

En lo que respecta a la dirección del sistema el proyecto creaba una Comisión Nacional de Educación, integrada por el Ministro de Gobierno, que la presidía, el Inspector Nacional de Educación como secretario, y además: el Rector de la Universidad, el director de la Escuela Normal del Estado, los Inspectores de Montevideo, Canelones y San José y cuatro miembros más, designados por el Inspector, de acuerdo con la Comisión: en total, 11 miembros. Las funciones serían de carácter general: selección de textos escolares, examen de maestros que aspiren al titulo, administración y dirección de la Escuela Normal, etc. Estimando Varela que él sería designado Inspector Nacional -tal como ocurrió- esta integración le aseguraba casi la mayoría para tomar decisiones, puesto que usualmente el Ministro de Gobierno no asistiría a las sesiones. Por otra parte, al ser éste un organismo lento, que sólo se reuniría cada tres meses, la dirección permanente quedaría en manos de Varela, como Inspector Nacional.

\section{La ley de educación común}

Si bien Varela contaba con el apoyo de Gobernador Latorre y del Ministro de Gobierno Montero, su proyecto fue enviado a estudio de una comisión, que le introdujo importantes modificaciones. En primer lugar, toda la estructura participativa que había concebido Varela no fue tenida en cuenta y en sustitución de la misma se estableció un régimen altamente centralizado. Francisco Berra señalará más tarde:

${ }^{24}$ VARELA, José Pedro. (1910): Óp., cit, Tomo II, Cap. XVII, p. 147. 
Se pasó de un extremo a otro, cuando lo más conveniente habría sido dar a las comisiones Departamentales alguna mayor ingerencia para lo cual pudiera ser suficiente el buen sentido y la influencia de los Inspectores como medio de acostumbrar a las poblaciones a manejar sus intereses escolares y de prepararlas para una descentralización gradual. Pero, es indudable, que de los dos extremos, era muy preferible el de la ley, porque es el que mejor se adapta al estado social del pais, a pesar de sus vicios ${ }^{25}$.

El sistema que estableció la Ley consistió en una Dirección General de Instrucción Pública compuesta por 7 miembros: el Ministro de Gobierno, el Inspector Nacional, el Director de la Escuela Normal y 4 vocales, todos designados por el Poder Ejecutivo y con las más amplias atribuciones: dirigir la instrucción primaria en todo el país, dirigir la Escuela Normal, nombrar o destituir maestros, seleccionar libros de texto, extender diplomas de maestro, etc. La descentralización se limitaba a la existencia de Comisiones Departamentales, integradas por el Inspector Departamental y 3 miembros designados por la Junta Económico- Administrativa respectiva.

Por otra parte, si bien se mantuvo la gratuidad de la enseñanza y el régimen obligatorio (aunque disminuyendo en algo las sanciones a los padres si estos no cumplían con su obligación), se cambió el criterio respecto a la educación religiosa: la enseñanza del catecismo sería obligatoria, aunque se admitiría que el padre que no estuviese de acuerdo pudiese retirar a su hijo durante esa enseñanza. En lo que respecta a los recursos para la educación, no se estableció nada, quedando estos librados a los que se otorgasen en cada presupuesto nacional.

A continuación, el Gobierno designó a Varela -como Inspector Nacional- y los cuatro vocales que integrarían la Comisión Nacional. De esos cuatro vocales, tres de ellos habían integrado la Sociedad de Amigos de la Educación Popular, junto con Varela, por lo que así parecía asegurada la continuidad de una filosofía educacional inaugurada una década atrás.

Contra lo que podría esperarse, Varela no opuso reparos a las modificaciones introducidas a su proyecto y se dedicó de lleno a poner en marcha el sistema, acorde con su pensamiento, según lo recordara más adelante Francisco Berra:

Se que mi actitud contribuye a prestigiar la dictadura, pero se también que si por este lado hago mal a mi país, por otro lado le hago bien. El prestigio que puedo dar a este gobierno es transitorio.

${ }^{25}$ BERRA, Francisco. (1888): Noticia de José Pedro Varela y de su participación en el reforma escolar del Uruguay. Buenos Aires. p. 65. 
El influjo de la reforma escolar es duradero y profundo. Peso en mi conciencia ambos hechos y no tengo la menor duda deque hago a mi país más bien que mal'2.

\section{Implementación de la reforma escolar: dificultades, avances}

Intensa fue la actividad de Varela en los meses subsiguientes a su designación: en primer lugar se designaron los Inspectores Departamentales; de los 14 que debían asumir sus cargos (dos por Montevideo y uno por cada Departamento del Interior) sólo 4 eran uruguayos; los demás eran: seis españoles, un argentino, un polaco, un italiano y un alemán, lo que muestra la escasez de personal calificado que existía en ese momento. Otro de los problemas que enfrentó Varela fue el de la descentralización: al reglamentarse la Ley, se previó que las Comisiones Departamentales de Educación (designadas por cada Junta Económico-Administrativa) designaran a su vez sub-comisiones en cada localidad que no fuese capital departamental, pero esas sub-comisiones muchas veces no pudieron constituirse por falta de vecinos calificados o simplemente no funcionaron por falta de interés en sus integrantes. Un Inspector Departamental señalaba en su informe anual: "Desgraciadamente poco resultado han dado las sub-comisiones de Instrucción Pública; ba habido sin duda bonrosas y recomendables excepciones, pero la generalidad de ellas no ban tomado interés por el fomento de las escuelas a su cargo, ni las ha visitado, ni, en muchos casos han llegado siquiera a constituirse".

En la lista de dificultades que tuvo que afrontar Varela, estaba la escasez de recursos. Al no existir rentas propias de la educación, el sistema debía sustentarse con lo que se le otorgase en el presupuesto nacional. Si bien las partidas mejoraron un poco en relación a los anteriores porcentajes destinados a la educación, de todas maneras el monto resultaba insuficiente. Sin embargo la nueva administración escolar supo administrar inteligentemente esos escasos recursos y logró mejoras sustanciales en el número de escuelas y en el volumen de la matrícula:

Cuando yo fui nombrado Director de Instrucción Pública, habia en las escuelas de Montevideo, 8000 alumnos, hay ahora 10000, y cuando se organizó la actual Dirección General, habia en Canelones 1500, hay ahora 2000, en la Colonia habla 600, hay 800, en Florida habia 500, hay 600... Así, los hechos prueban que lejos de disminuir, aumentó considerablemente el número de alumnos que asisten a las escuelas públicas ${ }^{27}$.

26 ACEVEDO, Edo. (1934): “Anales históricos del Uruguay”. Tomo IV. p. 97.

${ }^{27}$ Carta a: "EL SIGLO", sin fecha. (Archivo Museo pedagógico). 
En efecto, la matrícula escolar no solamente aumentó en los meses inmediatos, sino que continuó expandiéndose a intenso ritmo: al asumir el cargo en 1877, había 17.000 niños en las escuelas públicas y dos años después ya llegaban a 23.200 (un 36\% de aumento). Pero no fue sólo esa la transformación de la escuela pública: se mejoraron los programas y la metodología, los maestros se titularon en mayor número y, si bien no se había creado la Escuela Normal que proyectó Varela, se realizaban charlas sobre temas educativos de manera regular, muchas de ellas a cargo del propio Inspector Nacional, charlas a las que debían asistir obligatoriamente los maestros.

En este plano de la profesionalización del magisterio cabe incluir también la profesionalización del personal inspectivo, no ya a través de cursos, sino de reuniones de carácter técnico. Varela convocó y realizó el primer Congreso de Inspectores Departamentales en el año 1878, durante el cual se analizaron múltiples temas de naturaleza pedagógica: horarios, programas, métodos, etc.

Hubo, sin embargo, un tema que no llegó a resolver adecuadamente y éste fue el de la educación religiosa. Varela tuvo que aceptar que se enseñase religión en las escuelas y no hubo reacciones negativas por parte de los padres de alumnos, ya que la mayoría practicaban la religión católica y los otros quizás no deseaban generar un enfrentamiento con la Iglesia. Para amortiguar en algo la influencia de ésta en las escuelas públicas, los tiempos dedicados a la enseñanza religiosa fueron muy acotados; en el primer congreso de inspectores que ya mencionamos, se estableció que el tiempo que debía dedicarse a la asignatura "Moral y Religión" sería un 4\%, lo que para un máximo de 6 horas de clase, equivalía apenas a 15 minutos diarios, muy por debajo de otras asignaturas, tales como "Lecciones sobre Objetos" (alrededor de 70 minutos), "Geografía" (30 minutos). Esta actitud de las autoridades escolares generó protestas por parte de la prensa y de grupos de señoras católicas, frente a las cuales, Varela se defendió sosteniendo, en carta dirigida a un periódico: "Ni yo ni la Corporación que presido bemos pretendido ni pretendemos desconocer ni reformar ninguno de los artículos de la Constitución de la República, como no pretendemos tampoco contrariar el sentimiento religioso de los padres que envían sus bijos a la escuela pública”28.

Uno de los ideólogos de la enseñanza religiosa era Francisco Bauzá, quien sostenía:

En primer lugar conste que el país es católico por voluntad propia y por tradición histórica, en su absoluta mayoría.... En segundo lugar debe decirse también que la Constitución del Estado

\footnotetext{
${ }^{28}$ Carta sin fecha. (Archivo Mueso Pedagógico).
} 
declara culto oficial a la Religión Católica y no es por lo tanto extraño que esa religión tenga ciertas preeminencias sobre las otras que coexisten al lado de ella. ¿Cómo admirarse entonces de que los padres de familia y el clero católico pidan que en las escuelas públicas costeadas por el gobierno se enseñe a los niños la doctrina cristiana y se le haga rezar de entrada y de salida una sencilla oración pidiendo al Eterno el perdón de todos los hombres y el pan de todos los días? ${ }^{29}$.

No pudo Varela, sin embargo, calmar las inquietudes de la Iglesia Católica, que aspiraba a una más profunda influencia en el sistema público y ese enfrentamiento perduró mucho más allá de la actuación de aquel, prolongándose hasta principios del siglo XX en que por Ley de 1909 se suprime totalmente la enseñanza religiosa en las escuelas. En el aspecto que pudo Varela influir positivamente fue -sin duda- en el mejoramiento del personal docente, pero también en un cambio cualitativo que tendrá repercusión en la posterior evolución del sistema escolar: se trata de la feminización de ese personal, que -hasta la reforma escolar- estaba constituido mayoritariamente por hombres. Los cambios logrados gracias a los nuevos métodos y programas, que hacían menos violento el clima escolar, menos agresivo, posibilitó, sin duda, que muchas jóvenes se volcaran al magisterio, sobre todo cuando este se presentaba ordenado, con normas claras y con la posibilidad de desarrollar una carrera profesional. Esta feminización ayudó a que las autoridades escolares -primero José P. Varela, luego su hermano Jacobo- captase la ventaja económica de tener maestras en lugar de maestros, ya que las primeras podían trabajar por un sueldo menor que aquel que los hombres requerían normalmente:

El ejemplo de la mayor parte de los países civilizados y los hechos que se presentan a la más sencilla observación de nuestra actual organización social, demuestran cuan errónea es la idea de señalar un mismo sueldo a los maestros y maestras de igual categoría. No ignora Ud. que bajo el punto de vista económico, la oferta se regula por la demanda... Abora bien, por causas múltiples que no es del caso mencionar, el hombre tiene muchas más carreras y ocupaciones a que dedicarse con provecho, que la mujer..... En sus condiciones actuales, el magisterio es una carrera honrosa y lucrativa para la mujer, que no encuentra otras ocupaciones a que dedicarse en las que reciba mayor o igual retribución ${ }^{30}$.

Otras reformas introdujo Varela en los aspectos administrativos, como la graduación de los títulos que se expedían a los aspirantes, en función de

${ }^{29}$ BAUZA, Fco. (1879): De la educación común, p. 12.

${ }^{30}$ VARELA, José P. “Memoria correspondiente al período 1877-1878”, Cap.VI, en: VARELA, José Pedro. Tomo III, Cámara de Representantes- Repca. O. del Uruguay, p. 27. 
los conocimientos que se exigían en cada caso, adecuándose asimismo las retribuciones según el grado y responsabilidad que le cabía a cada educador. La buena administración financiera hizo posible que se comenzase a cumplir con el pago regular de los sueldos, lo que casi nunca había ocurrido; anteriormente se llegaban a registrar atrasos de seis o ocho meses, lo que convertía en indigna la profesión docente.

Un aspecto a señalar de la actuación de Varela al frente de las escuelas públicas, tiene que ver con su estilo directriz. Pese a la pregonada filosofía de amplia participación popular, Varela dirigió el sistema escolar en los escasos tres años en que lo hizo, de manera centralista y hasta autocrática. No acepto críticas a su labor, despidiendo a algún maestro si manifestaba su desacuerdo con las medidas tomadas, o presionando sutilmente a las Comisiones Departamentales para que actuaran de cierta manera. En una oportunidad, envía a una de esa Comisiones una nota en la que expresa:

lleva por cometido [quien actúa como delegado de Varela] el mismo que llevaba yo, esto es buscar los medios de que se modifique sustancialmente la constitución de la Comisión Departamental. He recibido seguridades de que si a tal cosa no pudiera llegarse buenamente, se nos prestarla el concurso necesario por quien puede hacerlo, para conseguirlo de otro medio... Al efecto, solicité y obtuve del Sr. Gobernador, carta que incluyo... El Sr. Álvarez... explicaráa usted las razones que demuestran la imposibilidad de marchar con la actual Comisión Departamental y a la vez el buen deseo de evitar que se resuelvan por actos de autoridad cuestiones de importancia que la Comisión... puede resolver sin eso ${ }^{31}$.

Es decir, Varela no duda en recurrir al autoritarismo del Cnel. Latorre para lograr sus objetivos y en otro caso, la presión es indirecta y sutil: pide en carta dirigida a su primo Mariano:

Querido Mariano: probablemente hoy o mañana se publicará el decreto nombrando Inspector Departamental de Instrucción Primaria y en cumplimiento de lo que dispone la ley, las Comisiones Extraordinarias deberán constituir las respectivas Comisiones Departamentales, que son presididas por un miembro de la Comisión Extraordinaria. Como se que en Canelones el Cura del Pueblo es Vicepresidente de la Comisión Extraordinaria y le gusta intervenir en las cuestiones de educación, temo que lo nombren Presidente de la Comisión Departamental de Instrucción Primaria y digo temo porque creo que puede ofrecer inconvenientes el que los curas sean Presidentes de las Comisiones de Instrucción Primaria. Por eso me ha ocurrido escribirte pidiéndote que si no tienes inconveniente

${ }^{31}$ Carta 26 de julio 1878. (Archivo Museo Pedagógico. Montevideo) 
influyas aunque sea indirectamente para que tal cosa no suceda. No querría sin embargo y por eso te escribo a ti particularmente, que esto llegara a conocimiento de la Comisión Extraordinaria de Canelones y del mismo cura porque en ese caso podría darse a este asunto una importancia que no tiene y atribuirme propósitos antirreligiosos que estoy lejos de abrigar en este caso... ${ }^{32}$.

Supo también Varela realizar una efectiva promoción de la reforma escolar que llevaba adelante mediante actos públicos que buscaban sensibilizar a la población. Así, a principios de 1879, realizó un concurso público entre las escuelas de Montevideo, otorgando premios a los escolares en un acto realizado en el Teatro Solis (el escenario de mayor prestigio en ese momento):

Los escolares, en número de 9000, divididos en 4 columnas y llevando al frente de cada una su respectiva banda de música, desfilaron ordenadamente por las calles más céntricas de Montevideo, ofreciendo un espectáculo tan hermoso como conmovedor, formando después en la plaza Independencia, desde la cual los alumnos premiados penetraban en el Teatro Solís, donde recibían la medalla o el diploma a que se habian becho acreedores... Hubo flores, versos, discursos a porfía, realizándose la fiesta hábilmente organizada por don Juan Alvarez y Pérez y presidida por don José M. Montero en representación del Gobierno... ${ }^{33}$.

\section{La muerte de Varela y la continuación de su proyecto}

A fines de 1879, Varela fallece a causa de una grave enfermedad que lo tuvo postrado con crueles sufrimientos durante sus últimos meses; en ese lapso, sin embargo, continuó dirigiendo el sistema escolar con empeño y dedicación, acelerando seguramente su final. Alfredo Vázquez Acevedo, en sus Memorias nos deja un cuadro dramático de sus últimos tiempos:

Todo el año 1879 lo pasamos preocupados con la saluda del pobre José Pedro. Una afección pertinaz al estómago (...) iba destruyéndolo día a día sin que se encontrara medio alguno de vencer el mal. Fueron llamados sucesivamente los mejores médicos, Méndez, Visca, Vidal, sin resultado alguno. Al fin, agobiado por la enfermedad, bizo cama; pero no se rindió. En el lecho trabajaba y atendía todas las tareas de la Dirección de Instrucción Primaria. Los dolores abatían su cuerpo, pero no doblaban su espíritu fuerte, carácter

\footnotetext{
${ }^{32}$ Carta a Mariano Berro. 19 de septiembre de 1877. (Archivo museo pedagógico)

33 ARAÚJO, Orestes. (1911): "Historia de la escuela uruguaya". Montevideo, Edit. El siglo ilustrado, p. 457.
} 
inquebrantable; se le veía muchas veces escribiendo con una mano y apretándose con la otra el vientre para calmar sus molestias. Todos lo acompañábamos durante las largas horas del día y de la noche. Yo, por mi parte, le dedicaba todo el tiempo que mis quehaceres me dejaban libre y le llevaba temas de conversación para distraerlo y hacerlo hablar... Pocos días después, el 24 a las 10 u 11 de la noche, falleció José Pedro Varela rodeado por su mujercita y por todos nosotros... ${ }^{34}$.

A su muerte, se habían alcanzado logros importantes en el sistema escolar: la matrícula escolar había crecido entre 1876 y 1879 de 17000 a más de 23000 alumnos; se crearon 100 nuevas escuelas, se titularon cientos de maestros, alcanzando estos un $75 \%$ del total, se publicaron las primeras estadísticas escolares, se realizó el primer congreso de Inspectores Escolares en el que se discutieron y aprobaron numerosas medidas respectos a programas, métodos, horarios, etc., la administración escolar fue cuidadosa y clara, permitiendo bajar el costo por escolar en un $22 \%$.

A penas tres años había estado al frente del sistema público, pero ya era reconocido como un insustituible artífice de ese sistema. Ello dio lugar a que su entierro convocara a una multitud de más de 20.000 personas que acompañaron su féretro por las calles de Montevideo. Resulta interesante considerar que entre quienes hicieron uso de la palabra en su entierro, estaba un representante de la Masonería Uruguaya, lo que reafirma la idea de la cercanía de Varela con esta organización.

Tal era el valor de la figura desaparecida en el cargo que desempeñaba, que el Gobierno del Cnel. Latorre hesitó al nombrar su sustituto, debiendo consultar a varias personalidades del medio. Al fin, fue elegido el hermano mayor de José Pedro, Jacobo. Este no era un experto en temas educacionales, pero había acompañado la obra de su hermano desde la época de la creación de la Sociedad de Amigos de la Educación Popular y tenía amplia experiencia como administrador, a partir de su actividad como comerciante.

No fue errada esta designación, ya que en los diez años que ejerció su cargo de Inspector Nacional de Instrucción Primaria, realizó una fecunda obra que continuó y amplió la obra de su hermano: convocó un segundo congreso de Inspectores Departamentales, sistematizó la información estadística, creó el "Internato Normal de Señoritas" para la formación regular de las maestras, extendió el número de escuelas rurales, fundó el Museo y Biblioteca Pedagógico, que -hasta hoy- es ejemplo en la región, etc.

34 VÁZQUEZ ACEVEDO, A. (1965): “Memoria”. Tomo. VII. Uruguay, Montevideo, editorial: A. Barreiro y Ramos. 
A la finalización de su mandato en 1889, el número de escuelas y de alumnos había llegado a más de 400 y más de 32.000; se había consolidado el profesionalismo de la función docente y todo el sistema escolar se encontraba en franco desarrollo.

\section{CONCLUSIÓN}

Surge de lo expuesto anteriormente, que José Pedro Varela merece ser considerado entre los educadores latinoamericanos destacados. No tuvo -sin embargo- reconocimientos fuera de fronteras en las primeras décadas de su muerte; por ejemplo, el Diccionario de Pedagogía Labor, en edición de 1936 no dedica ninguna página a Varela y su obra, en tanto dedica varias a otros educadores del concierto latinoamericano del siglo XIX, como Faustino Sarmiento, Gabino Barreda, Enrique José Varona, José de la Luz y Caballero, Alfredo Miguel Aguayo, etc.

Ciertamente no ejerció Varela influencia sobre la región -seguramente por su corta vida- pero sí sobre su país. En la segunda mitad del siglo XIX Uruguay no contaba con un sistema escolar mínimo: apenas funcionaban pocas escuelas sin coordinación entre sí, sin métodos pedagógicos, sin textos, con maestros sin formación específica y muchas veces cercanos a la ignorancia. Fue a partir de 1868 que esa situación comenzó a ser cuestionada firmemente por Varela y el núcleo de jóvenes que lo acompañaron en la Sociedad de Amigos de la Educación Popular: creando escuelas, bibliotecas populares, editando libros, organizando conferencias. A partir de ahí el sistema escolar uruguayo se asentó firmemente sobre las bases que el joven Varela impulsó y sostuvo: educación universal, obligatoria, gratuita y laica.

Cierto es que algunas de estas ideas no fueron aceptadas en su momento -la laicidad se implantó totalmente años después de su muerte y la participación popular recién hoy está comenzando a instrumentarse- pero su pensamiento y su obra han constituido hasta hoy un norte claro para los educadores uruguayos. Tan es así que su obra -la "Reforma Escolar"se ha llegado a constituir casi en un mito, que ha distorsionado la clara comprensión del proceso educacional uruguayo al sobredimensionar el papel de José Pedro Varela (desconociendo casi el de su hermano): se han tomado algunos pocos elementos de su vida y su obra y sobre ellos se ha construido una tradición que lo ha convertido en un personaje casi sobre-humano, capaz por sí solo de construir el sistema escolar nacional, enfrentando un sin fin de enemigos y oposiciones. Este enfoque va a contrapelo de un análisis histórico serio que no reconoce las acciones individuales como único elemento de transformación social. 


\section{FUENTES}

VARELA, José P. (1876-1877): Memoria de la Dirección General de Instrucción Primaria.

VARELA, José P. Memoria correspondiente al período transcurrido desde el 24 de agosto de 1877 al 31 de octubre de 1878, presentada a la Dirección de Instrucción Pública.

VARELA, Jacobo: Memoria correspondiente a los años 1879-1880, presentada a la Dirección de Instrucción Pública por el Inspector Nacional de Instrucción Pública.

VARELA, José Pedro. (1874): La Educación del Pueblo. Uruguay, Edit. Sociedad de Amigos de la Educación Popular.

VARELA, José Pedro. (1875): La Legislación Escolar. Uruguay, editorial Montevideo, Edit. Sociedad de Amigos de la Educación Popular.

VARELA, José Pedro. (1945): Impresiones de viaje a Europa y Estados Unidos. Uruguay. Edit. Liceo. Montevideo.

VARELA, José Pedro; RAMÍREZ, Carlos M. (1964): El destino nacional y la Universidad: polémica. Ministerio de Instrucción Pública y Previsión Social. Editorial Montevideo.

VÁZQUEZ ROMERO, A. (1979): José P. Varela. Editorial Montevideo, Casa del Estudiante.

\section{REFERENCIAS}

ARAÚJO, Orestes. (1911): Historia de la Escuela Uruguaya. Editorial Montevideo, Imp. "El Siglo ilustrado".

BECERRO DE BENGOA, Julián. (1908): La Reforma Vareliana en Campaña (en "Obras de José Pedro Varela" Tomo III- Cámara de RepresentantesRepca. O. del Uruguay.

BERRA, Francisco A. (1888): Noticia sobre José P. Varela y su participación en la reforma escolar del Uruguay. Uruguay, editorial, Buenos Aires.

BRALICH, Jorge. (1989): Varela. Sociedad Burguesa y Reforma Educacional. Montevideo, Ediciones del Nuevo Mundo.

GONZÁLEZ ALBISTUR, Jorge. (1997): José Pedro Varela. Montevideo, Uruguay, Ediciones de la Plaza.

HERRERO Y ESPINOSA, Miguel. (1885): José Pedro Varela, Montevideo, Edit. Lagomarsino. 
LASPLACES, Alberto. (1928): Vida admirable de José P. Varela, Montevideo.

LERENA DE BLIXEN, Josefina: (1948): Varela, el reformador. Uruguay, editorial Montevideo. [s.n.]

MANACORDA, Telmo. (1945): José Pedro Varela. Uruguay, editorial Montevideo, Impr. Uruguaya.

MONESTIER, Jaime. (1992): El combate laico- bajorrelieve de la reforma vareliana. Uruguay, editorial Montevideo, Ediciones El Galeón.

MONTERO BUSTAMANTE, Raúl. (1922): Jacobo A. Varela: Su vida y su obra. Editorial. Montevideo: [s.n.].

PERDOMO CORONEL, Nazareth. (1945): José Pedro Varela, Ideario. Montevideo, ditorial Mundo Libre.

BRALICH, Jorge. (2011): "José Pedro Varela y la gestación de la escuela uruguaya" en: Revista Historia de la Educación Latinoamericana Vol. 13 No. 17, Tunja, Universidad Pedagógica y Tecnológica de Colombia, RUDECOLOMBIA, SHELA- HISULA. pp. $43-70$ 